\title{
Fuzzy Szpilrajn Theorem through Indicators
}

\author{
Irina Georgescu
}

\begin{abstract}
In this paper there are studied some numerical indicators which measure the degree to which a fuzzy relation verifies some properties (reflexivity, transitivity, etc. ).The main result is a fuzzy generalization of the Szpilrajn theorem in terms of such numerical indicators and is applied to any fuzzy relation.

Keywords: fuzzy relation, Szpilrajn theorem, similarity
\end{abstract}

\section{Introduction}

The classical Szpilrajn theorem [10] asserts that any partial order can be extended to a total order. This result has been followed by refinements and generalizations and has been used in applications too (e. g. consumer theory [8]).

The first fuzzy version of the Szpilrajn theorem has been established by Zadeh [11]. Other fuzzy versions of this theorem can be found in [4], [6]. In [3] the topic is systematically studied in the framework of the fuzzy orders with respect to a left-continuous t-norm $*$ and a $*$-similarity relation $\Omega$.

The idea of this paper is the following: instead of studying a property $P$ of a fuzzy relation $R$ (e. g. reflexivity, transitivity, etc.) to define numerical indicators which should express "the degree to which the fuzzy relation $R$ verifies the property $P^{\prime \prime}$. In this way, instead of considering a fuzzy order $R$ on a set $X$ we will have a number $\operatorname{Ord}(R)$ which should measure "the degree to which $R$ is a fuzzy order".

The main result of the paper is a generalization of the Szpilrajn theorem expressed in terms of such numerical indicators. It is a refinement of Theorem 6.2 in [3] and it is applied to any fuzzy relation.

\section{Preliminaries}

In this section we shall recall some basic facts on the residuum associated with a left-continuous t-norm and on fuzzy relations ([1], [2], [4], [5], [7], [9]).

For any $a, b \in[0,1]$ we denote $a \vee b=\max (a, b)$ and $a \wedge b=\min (a, b)$. More generally, for any set $\left\{a_{i}\right\}_{i \in I} \subseteq[0,1]$ we denote $\bigvee_{i \in I} a_{i}=\sup \left\{a_{i} \mid i \in I\right\}$ and $\bigwedge_{i \in I} a_{i}=\inf \left\{a_{i} \mid i \in I\right\}$.

Let $*$ be a left-continuous $\mathrm{t}-$ norm [7], [5]. The residuum $\rightarrow$ associated with $*$ is introduced by

$a \rightarrow b=\bigvee\{c \in[0,1] \mid a * c \leq b\}$.

The biresiduum $\leftrightarrow$ is denoted by $a \leftrightarrow b=(a \rightarrow b) \wedge(b \rightarrow a)$.

We fix a left-continuous t-norm $*$.

Lemma 1. [1], [5] For any $a, b, c \in[0,1]$ the following properties hold:

(1) $a * b \leq c$ iff $a \leq b \rightarrow c$;

(2) $a \wedge b=a *(a \rightarrow b)$;

(3) $a \leq b$ iff $a \rightarrow b=1$;

(4) $a=1 \rightarrow a$;

(5) $1=a \rightarrow a$;

(6) $a \leq a *(a \leftrightarrow b)$. 
Lemma 2. [1], [5] For any $\left\{a_{i}\right\}_{i \in I} \subseteq[0,1]$ and $a \in[0,1]$ the following properties hold:

(1) $\left(\bigvee_{i \in I} a_{i}\right) * a=\bigvee_{i \in I}\left(a_{i} * a\right)$;

(2) $a \rightarrow\left(\bigwedge_{i \in I} a_{i}\right)=\bigwedge_{i \in I}\left(a \rightarrow a_{i}\right)$;

(3) $\left(\bigvee_{i \in I} a_{i}\right) \rightarrow a=\bigwedge_{i \in I}\left(a_{i} \rightarrow a\right)$.

Let $X$ be a non-empty subset. A fuzzy subset of $X$ is a function $A: X \rightarrow[0,1]$. Denote by $\mathcal{F}(X)$ the family of the fuzzy subsets of $X$. For any $A, B \in \mathcal{F}(X)$ denote $A \subseteq B$ if $A(x) \leq B(x)$ for any $x \in X$.

A fuzzy relation on $X$ is a function $R: X^{2} \rightarrow[0,1] . R$ is said to be

- reflexive if $R(x, x)=1$ for any $x \in X$;

- symmetric if $R(x, y)=R(y, x)$ for any $x, y \in X$;

- $*$-transitive if $R(x, y) * R(y, z) \leq R(x, z)$ for all $x, y, z \in X$;

- strongly complete if $R(x, y) \vee R(y, x)=1$ for any $x, y \in X$.

A reflexive, symmetric and $*$-transitive fuzzy relation $\Omega$ on $X$ will be called $*$-similarity relation.

Let $\Omega$ be a $*$-similarity relation on $X$ and $R$ a fuzzy relation on $X$. $R$ is said to be:

- $\Omega$-reflexive if $\Omega(x, y) \leq R(x, y)$ for any $x, y \in X$;

- $(*, \Omega)$-antisymmetric if $R(x, y) * R(y, x) \leq \Omega(x, y)$ for any $x, y \in X$.

A $(*, \Omega)$-order is $*$-transitive, $\Omega$-reflexive and $(*, \Omega)$-antisymmetric fuzzy relation $R$ on $X$. Let $R, Q$ be two $(*, \Omega)$-orders on $X$. We say that $Q$ is an extension of $R$ if $R \subseteq Q$, i. e. $R(x, y) \leq Q(x, y)$ for all $x, y \in X$.

The following fuzzy generalization of the Szpilrajn theorem was proved in [3]:

Theorem 3. Let $\Omega$ be a $*$-similarity relation on $X$. Then any $(*, \Omega)$-order on $X$ has a strongly complete extension.

\section{Some indicators}

Let $*$ be a left-continuous $\mathrm{t}-$ norm and $\Omega$ be a $*$-similarity relation on $X$.

Definition 4. For any fuzzy relation $R$ on $X$ let us define:

$$
\begin{aligned}
& \operatorname{Ref}(R)=\bigwedge_{x \in X} R(x, x) ; \\
& \operatorname{Trans}(R)=\bigwedge_{x, y, z \in X}[R(x, y) * R(y, z) \rightarrow R(x, z)] ; \\
& \operatorname{Ref}_{\Omega}(R)=\bigwedge_{x, y \in X}(\Omega(x, y) \rightarrow R(x, y)] ; \\
& \operatorname{Ant}_{\Omega}(R)=\bigwedge_{x, y \in X}[R(x, y) * R(y, x) \rightarrow \Omega(x, y)] ; \\
& S C(R)=\bigwedge_{x, y \in X}(R(x, y) \vee R(y, x)) ; \\
& \operatorname{Ord}_{\Omega}(R)=\operatorname{Re} f_{\Omega}(R) \wedge \operatorname{Ant}_{\Omega}(R) \wedge \operatorname{Trans}(R) .
\end{aligned}
$$

Lemma 5. For any fuzzy relation $R$ the following equivalences hold:

(1) $\operatorname{Ref}(R)=1$ iff $R$ is reflexive;

(2) $\operatorname{Trans}(R)=1$ iff $R$ is $*$-reflexive; 
(3) $\operatorname{Re}_{\Omega}(R)=1$ iff $R$ is $\Omega$-reflexive;

(4) $\operatorname{Ant}_{\Omega}(R)=1$ iff $R$ is $(*, \Omega)$-antisymmetric;

(5) $S C(R)=1$ iff $R$ is strongly complete;

(6) $\operatorname{Ord}_{\Omega}(R)=1$ iff $R$ is a $(*, \Omega)$-order.

$\operatorname{Re} f(R)$ will be called the degree of reflexivity of $R, \operatorname{Trans}(R)$ the degree of $*$-transitivity of $R$, etc. The indicators introduced above refine the properties of reflexivity, transitivity, etc. of fuzzy relations. Thus, instead of saying that the fuzzy relation $R$ is reflexive, the real number $\operatorname{Re} f(R)$ will measure "the degree to which $R$ is reflexive".

Proposition 6. Let $R$ be a fuzzy relation on $X$ and $x, y, z \in X$. Then

(1) $\operatorname{Ref}(R) \leq R(x, x)$;

(2) $\operatorname{Trans}(R) * R(x, y) * R(y, z) \leq R(x, z)$;

(3) $\operatorname{Ref}_{\Omega}(R) * \Omega(x, y) \leq R(x, y)$;

(4) $\operatorname{Ant}_{\Omega}(R) * R(x, y) * R(y, x) \leq \Omega(x, y)$;

(5) $\operatorname{Ord}_{\Omega}(R) \leq \Omega(x, y) \leftrightarrow(R(x, y) * R(y, x))$;

(6) $\operatorname{Ord}_{\Omega}(R) \leq R(x, x)$.

\section{Main result}

In this section we shall prove a generalization of the theorem of Szpilrajn formulated in terms of the indicators introduced in the previous paragraph. The result will be valid for any fuzzy relations and in particular one will obtain Theorem 3.

Let $*$ be a left-continuous t-norm and $\Omega$ a $*$-similarity relation on $X$. If $R$ and $Q$ are two fuzzy relations on $X$ then we denote

$R \preccurlyeq Q$ iff $R \subseteq Q$ and $\operatorname{Ord}_{\Omega}(R) \leq \operatorname{Ord}_{\Omega}(Q)$.

It is easy to see that $\preccurlyeq$ is a partial order on the set of the fuzzy relations defined on $X$. If $Q$ is a fuzzy relation on $X$ then we denote by $\operatorname{Ext}(Q)$ the set of all fuzzy relations $R$ on $X$ with the property that $Q \preccurlyeq R$.

Lemma 7. If $Q$ is a fuzzy relation on $X$ then the partially ordered $\operatorname{set}(\operatorname{Ext}(Q), \preccurlyeq)$ admits a maximal element.

Proof. We prove that $(\operatorname{Ext}(Q), \preccurlyeq)$ is inductive. We consider a chain $\left(R_{i}\right)_{i \in I}$ in $\operatorname{Ext}(Q)$ : for any $i, j \in I$ we have $R_{i} \preccurlyeq R_{j}$ or $R_{j} \preccurlyeq R_{i}$. Of course $Q \preccurlyeq R_{i}$ for any $i \in I$. We will denote $R=\bigcup_{i \in I} R_{i}$. It suffices to prove that $R \in \operatorname{Ext}(Q)$. It is obvious that $Q \subseteq R$ therefore we have to prove that $\operatorname{Ord}_{\Omega}(Q) \leq \operatorname{Ord}_{\Omega}(R)$. We show first that

(a) $\operatorname{Ord}_{\Omega}(Q) \leq \operatorname{Ref}_{\Omega}(R)$.

Let $x, y \in X$ and $i \in I$. Since $Q \subseteq R_{i}$ it follows immediately

$\operatorname{Ord}_{\Omega}(Q) \leq \operatorname{Re}_{\Omega}(Q) \leq \operatorname{Re}_{\Omega}\left(R_{i}\right) \leq \Omega(x, y) \rightarrow R_{i}(x, y)$.

By applying Lemma 1 (2) and the previous inequality

$\operatorname{Ord}_{\Omega}(Q) * \Omega(x, y) \leq \Omega(x, y) *\left(\Omega(x, y) \rightarrow R_{i}(x, y)\right)=$

$=\Omega(x, y) \wedge R_{i}(x, y) \leq R_{i}(x, y) \leq R(x, y)$

from where according to Lemma $1(1), \operatorname{Ord}_{\Omega}(Q) \leq \Omega(x, y) \rightarrow R(x, y)$. It follows 
$\operatorname{Ord}_{\Omega}(Q) \leq \bigwedge_{x, y \in X}(\Omega(x, y) \rightarrow R(x, y))=\operatorname{Ref}_{\Omega}(R)$.

We intend to prove that

(b) $\operatorname{Ord}_{\Omega}(Q) \leq A n t_{\Omega}(R)$

Let $x, y \in X$. Then

$\operatorname{Ord}_{\Omega}(Q) * R(x, y) * R(y, x)=\operatorname{Ord}_{\Omega}(Q) *\left[\bigvee_{i \in I} R_{i}(x, y)\right] *\left[\bigvee_{j \in I} R_{j}(y, x)\right]=$

$=\bigvee_{i, j \in I} \operatorname{Ord}_{\Omega}(Q) * R_{i}(x, y) * R_{j}(y, x)$.

Let $i, j \in I$. Assume that $R_{i} \preccurlyeq R_{j}$ therefore $R_{i} \subseteq R_{j}$ and $\operatorname{Ord}_{\Omega}\left(R_{i}\right) \leq \operatorname{Ord}_{\Omega}\left(R_{j}\right)$. Then, according to $Q \preccurlyeq R_{j}$ and Proposition $6(4)$ :

$\operatorname{Ord}_{\Omega}(Q) * R_{i}(x, y) * R_{j}(y, x) \leq \operatorname{Ord}_{\Omega}\left(R_{j}\right) * R_{j}(x, y) * R_{j}(y, x) \leq$

$\leq A n t_{\Omega}\left(R_{j}\right) * R_{j}(x, y) * R_{j}(y, x) \leq \Omega(x, y)$.

Since this inequality is valid for any $i, j \in I$ it follows $\operatorname{Ord}_{\Omega}(Q) * R(x, y) * R(y, x) \leq \Omega(x, y)$, therefore, according to Lemma $1(1), \operatorname{Ord}_{\Omega}(Q) \leq R(x, y) * R(y, x) \rightarrow \Omega(x, y)$. From here we deduce

$\operatorname{Ord}_{\Omega}(Q) \leq \bigwedge_{x, y \in X}[R(x, y) * R(y, x) \rightarrow \Omega(x, y)]=\operatorname{Ant}_{\Omega}(R)$.

We still have to prove

(c) $\operatorname{Ord}_{\Omega}(Q) \leq \operatorname{Trans}(R)$.

Let $x, y \in X$. Then

$\operatorname{Ord}_{\Omega}(Q) * R(x, y) * R(y, z)=\bigvee_{i, j \in I} \operatorname{Ord}_{\Omega}(Q) * R_{i}(x, y) * R_{j}(y, x)$

Let $i, j \in I$. Assume $R_{i} \preccurlyeq R_{j}$ therefore $R_{i} \subseteq R_{j}$ and $\operatorname{Ord}_{\Omega}\left(R_{i}\right) \leq \operatorname{Ord}_{\Omega}\left(R_{j}\right)$. According to Proposition $6(2)$ :

$\operatorname{Ord}_{\Omega}(Q) * R_{i}(x, y) * R_{j}(y, z) \leq \operatorname{Ord}_{\Omega}\left(R_{j}\right) * R_{j}(x, y) * R_{j}(y, z) \leq$

$\leq \operatorname{Trans}\left(R_{j}\right) * R_{j}(x, y) * R_{j}(y, z) \leq R_{j}(x, z) \leq R(x, z)$

from where, $\operatorname{Ord}_{\Omega}(Q) * R(x, y) * R(y, z) \leq R(x, z)$. By applying Lemma 1 (1) it follows that for any $x, y, z \in X$ we have $\operatorname{Ord}_{\Omega}(Q) \leq R(x, y) * R(y, z) \rightarrow R(x, z)$, from where

$\operatorname{Ord}_{\Omega}(Q) \leq \bigwedge_{x, y, z \in X}[R(x, y) * R(y, z) \rightarrow R(x, z)]=\operatorname{Trans}(R)$.

From (a), (b) and (c) one obtains $\operatorname{Ord}_{\Omega}(Q) \leq \operatorname{Ord}_{\Omega}(R)$. We have shown that $(\operatorname{Ext}(Q), \preccurlyeq)$ is inductive. According to Zorn's axiom a maximal element exists in $\operatorname{Ext}(Q)$.

In the following we will situate ourselves in the case of the Gödel t-norm $\wedge$.

Theorem 8. Let $Q$ be a fuzzy relation on $X$. Then there exists a fuzzy relation $R$ on $X$ such that $Q \preccurlyeq R$ and $\operatorname{Ord}_{\Omega}(Q) \leq S C(R)$.

Proof. According to Lemma 7 there exists a fuzzy relation $R$ on $X$ maximal in $(\operatorname{Ext}(Q), \preccurlyeq)$. Then $Q \preccurlyeq R$. It remains to prove that $\operatorname{Ord}_{\Omega}(Q) \leq S C(R)$. We assume by absurdum that

$\operatorname{Ord}_{\Omega}(Q) \not \leq S C(R)=\bigwedge_{x, y \in X}(R(x, y) \vee R(y, x))$.

therefore there exist $a, b \in X$ such that $\operatorname{Ord}_{\Omega}(Q) \not \leq R(a, b) \vee R(b, a)$ from where $\operatorname{Ord}_{\Omega}(Q) \not \leq R(a, b)$ and $\operatorname{Ord}_{\Omega}(Q) \not \leq R(b, a)$. Assume $R(a, b) \leq R(b, a)$. According to the lines above, $R(b, a)<\operatorname{Ord}_{\Omega}(Q)$. We define a new fuzzy relation $R^{\prime}$ on $X$ by

$R^{\prime}(x, y)=R(x, y) \vee(R(x, b) \wedge R(a, y))$

for any $x, y \in X$. We intend to prove that $R \preccurlyeq R^{\prime}$. It is obvious that $R \subseteq R^{\prime}$ therefore it remains to prove that $\operatorname{Ord}_{\Omega}(R) \leq \operatorname{Ord}_{\Omega}\left(R^{\prime}\right)$. From $R \subseteq R^{\prime}$ it follows immediately

(1) $\operatorname{Ord}_{\Omega}(R) \leq \operatorname{Re} f_{\Omega}\left(R^{\prime}\right)$

We establish now the inequality:

(2) $\operatorname{Ord}_{\Omega}(R) \leq A n t_{\Omega}\left(R^{\prime}\right)$

Let $x, y \in X$. Then 
$\operatorname{Ord}_{\Omega}(R) \wedge R^{\prime}(x, y) \wedge R^{\prime}(y, x)=$

$=\operatorname{Ord}_{\Omega}(R) \wedge[R(x, y) \vee(R(x, b) \wedge R(a, y))] \wedge[R(y, x) \vee(R(y, b) \wedge R(a, x))]=$

$=\left[\operatorname{Ord}_{\Omega}(R) \wedge R(x, y) \wedge R(y, x)\right] \vee\left[\operatorname{Ord}_{\Omega}(R) \wedge R(x, y) \wedge R(y, b) \wedge R(a, x)\right] \vee$

$\vee\left[\operatorname{Ord}_{\Omega}(R) \wedge R(y, x) \wedge R(x, b) \wedge R(a, y)\right] \vee\left[\operatorname{Ord}_{\Omega}(R) \wedge R(x, b) \wedge R(a, y) \wedge R(y, b) \wedge R(a, x)\right]$

We will establish the following inequalities:

(a) $\operatorname{Ord}_{\Omega}(R) \wedge R(x, y) \wedge R(y, x) \leq \Omega(x, y)$;

(b) $\operatorname{Ord}_{\Omega}(R) \wedge R(x, y) \wedge R(y, b) \wedge R(a, x) \leq \Omega(x, y)$;

(c) $\operatorname{Ord}_{\Omega}(R) \wedge R(y, x) \wedge R(x, b) \wedge R(a, y) \leq \Omega(x, y)$;

(d) $\operatorname{Ord}_{\Omega}(R) \wedge R(x, b) \wedge R(a, y) \wedge R(y, b) \wedge R(a, x) \leq \Omega(x, y)$.

In order to obtain (a) we use Proposition 6 (4):

$\operatorname{Ord}_{\Omega}(R) \wedge R(x, y) \wedge R(y, x) \leq A n t_{\Omega}(R) \wedge R(x, y) \wedge R(y, x) \leq \Omega(a, b)$.

We treat now the other three cases. According to Proposition 6 (5) we have

$\operatorname{Ord}_{\Omega}(R) \leq \Omega(a, b) \leftrightarrow(R(a, b) \wedge R(b, a))=\Omega(a, b) \leftrightarrow R(a, b)$

We consider first the case $R(x, y) \leq R(y, x)$. Then $\operatorname{Ord}_{\Omega}(R) \leq \Omega(x, y) \leftrightarrow R(x, y)$ with the same argument as above. (b) results like this:

$\operatorname{Ord}_{\Omega}(R) \wedge R(x, y) \wedge R(y, b) \wedge R(a, x) \leq R(x, y) \wedge[\Omega(x, y) \leftrightarrow R(x, y)] \leq \Omega(x, y)$

Now we treat cases (c) and (d). First we notice that according to Proposition 6 (2):

$\operatorname{Ord}_{\Omega}(R) \wedge R(y, x) \wedge R(x, b) \wedge R(a, y) \leq \operatorname{Trans}(R) \wedge R(a, y) \wedge R(y, x) \wedge R(x, b) \leq R(a, b)$

therefore

$\operatorname{Ord}_{\Omega}(R) \wedge R(y, x) \wedge R(a, y) \leq R(a, b) \wedge[R(a, b) \leftrightarrow \Omega(a, b)] \leq \Omega(a, b)$.

Analogously we obtain:

$\operatorname{Ord}_{\Omega}(R) \wedge R(x, b) \wedge R(a, y) \wedge R(y, b) \wedge R(a, x) \leq \Omega(a, b)$.

We consider the possible subcases:

(i) $\Omega(a, b) \leq R(x, y)$;

(ii) $\Omega(a, b)>R(x, y)$.

According to the proof above, in case (i) the inequalities (c) and (d) are immediate. We are situated now in case (ii). One notices that

$\operatorname{Ord}_{\Omega}(R) \wedge R(x, b) \wedge \Omega(a, b) \wedge R(a, y) \leq \Omega(a, b) \wedge[\Omega(a, b) \leftrightarrow R(a, b)] \leq R(a, b) \leq R(b, a)$

therefore

$\operatorname{Ord}_{\Omega}(R) \wedge R(x, b) \wedge \Omega(a, b) \wedge R(a, y) \leq \operatorname{Ord}_{\Omega}(R) \wedge R(x, b) \wedge R(b, a) \wedge R(a, y) \leq \operatorname{Trans}(R) \wedge R(x, b) \wedge$ $R(b, a) \wedge R(a, y) \leq R(x, y)$

From $\operatorname{Ord}_{\Omega}(R) \wedge R(x, b) \wedge \Omega(a, b) \wedge R(a, y) \leq R(x, y)$ and $\Omega(a, b)>R(x, y)$ it follows

$\operatorname{Ord}_{\Omega}(R) \wedge R(x, b) \wedge R(a, y) \leq R(x, y)$.

By using this last inequality we have

$\operatorname{Ord}_{\Omega}(R) \wedge R(y, x) \wedge R(x, b) \wedge R(a, y) \leq \operatorname{Ord}_{\Omega}(R) \wedge R(x, b) \wedge R(a, y) \leq R(x, y)$

from where

$\operatorname{Ord}_{\Omega}(R) \wedge R(y, x) \wedge R(x, b) \wedge R(a, y) \leq R(x, y) \wedge[R(x, y) \leftrightarrow \Omega(x, y)] \leq \Omega(x, y)$

Thus (c) was proved and (d) follows analogously.

The case $R(y, x) \leq R(x, y)$ is treated analogously. Therefore the inequalities (a)-(d) are true, so $\operatorname{Ord}_{\Omega}(R) \wedge R^{\prime}(x, y) \wedge R^{\prime}(y, x) \leq \Omega(x, y)$. Cf. Lemma 1 (1) for any $x, y, z \in X$ we have $\operatorname{Ord}_{\Omega}(R) \leq$ $\left(R^{\prime}(x, y) \wedge R^{\prime}(y, x)\right) \rightarrow \Omega(x, y)$ therefore

$\operatorname{Ord}_{\Omega}(R) \leq \bigwedge_{x, y \in X}\left[\left(R^{\prime}(x, y) \wedge R^{\prime}(y, x)\right) \rightarrow \Omega(x, y)\right]=A n t_{\Omega}\left(R^{\prime}\right)$

Now we establish the inequality

(3) $\operatorname{Ord}_{\Omega}(R) \leq \operatorname{Trans}\left(R^{\prime}\right)$.

Let $x, y, z \in X$. We prove

(4) $\operatorname{Ord}_{\Omega}(R) \wedge R^{\prime}(x, y) \wedge R^{\prime}(y, z) \leq R^{\prime}(x, z)$.

We notice that

$\operatorname{Ord}_{\Omega}(R) \wedge R^{\prime}(x, y) \wedge R^{\prime}(y, z)=$ 
$=\operatorname{Ord}_{\Omega}(R) \wedge[R(x, y) \vee(R(x, b) \wedge R(a, y))] \wedge[R(y, z) \vee(R(y, b) \wedge R(a, z))]=$
$=\left[\operatorname{Ord}_{\Omega}(R) \wedge R(x, y) \wedge R(y, z)\right] \vee\left[\operatorname{Ord}_{\Omega}(R) \wedge R(x, y) \wedge R(y, b) \wedge R(a, z)\right] \vee$
$\vee\left[\operatorname{Ord}_{\Omega}(R) \wedge R(y, z) \wedge R(x, b) \wedge R(a, y)\right] \vee\left[\operatorname{Ord}_{\Omega}(R) \wedge R(x, b) \wedge R(a, y) \wedge R(y, b) \wedge R(a, z)\right]$.

Then to prove (4) is equivalent with establishing the following inequalities:

(e) $\operatorname{Ord}_{\Omega}(R) \wedge R(x, y) \wedge R(y, z) \leq R^{\prime}(x, z)$;

(f) $\operatorname{Ord}_{\Omega}(R) \wedge R(x, y) \wedge R(y, b) \wedge R(a, z) \leq R^{\prime}(x, z)$;

(g) $\operatorname{Ord}_{\Omega}(R) \wedge R(y, z) \wedge R(x, b) \wedge R(a, y) \leq R^{\prime}(x, z)$;

(h) $\operatorname{Ord}_{\Omega}(R) \wedge R(x, b) \wedge R(a, y) \wedge R(y, b) \wedge R(a, z) \leq R^{\prime}(x, z)$.

(e) follows by applying Proposition $6(2)$ :

$\operatorname{Ord}_{\Omega}(R) \wedge R(x, y) \wedge R(y, z) \leq \operatorname{Trans}(R) \wedge R(x, y) \wedge R(y, z) \leq R(x, z) \leq R^{\prime}(x, z)$.

(f) and (g) follow like this:

$\operatorname{Ord}_{\Omega}(R) \wedge R(x, y) \wedge R(y, b) \wedge R(a, z) \leq(\operatorname{Trans}(R) \wedge R(x, y) \wedge R(y, b)) \wedge R(a, z) \leq R(x, b) \wedge R(a, z) \leq$ $R^{\prime}(x, z)$;

$\operatorname{Ord}_{\Omega}(R) \wedge R(y, z) \wedge R(x, b) \wedge R(a, y) \leq(\operatorname{Trans}(R) \wedge R(a, y) \wedge R(y, z)) \wedge R(x, b) \leq R(x, b) \wedge R(a, z) \leq$ $R^{\prime}(x, z)$.

(h) follows similarly. We established (e)-(h), therefore (4) is true. Cf. Lemma 1 (1) for any $x, y, z \in X$ we have $\operatorname{Ord}_{\Omega}(R) \leq\left(R^{\prime}(x, y) \wedge R(y, z)\right) \rightarrow R^{\prime}(x, z)$, from where

$\operatorname{Ord}_{\Omega}(R) \leq \bigwedge_{x, y, z \in X}\left[\left(R^{\prime}(x, y) \wedge R^{\prime}(y, z)\right) \rightarrow R^{\prime}(x, z)\right]=\operatorname{Trans}\left(R^{\prime}\right)$

From (1), (2) and (3) we deduce $\operatorname{Ord}_{\Omega}(R) \leq \operatorname{Ord}_{\Omega}\left(R^{\prime}\right)$ therefore $R \preccurlyeq R^{\prime}$. We can see that

$R^{\prime}(a, b)=R(b, a) \vee(R(b, b) \wedge R(a, a))$ and $R(b, a)<\operatorname{Ord}_{\Omega}(Q) \leq \operatorname{Ord}_{\Omega}(R)$ (since $Q \preccurlyeq R$ ). Then, by applying Proposition $6(6)$ :

$R(b, a)<\operatorname{Ord}_{\Omega}(R) \leq R(b, b) \wedge R(a, a) \leq R^{\prime}(a, b)$.

It follows $R \neq R^{\prime}$, contradicting the maximality of $R$. We conclude $\operatorname{Ord}_{\Omega}(Q) \leq S C(R)$, therefore the theorem is proved.

Remark 9. By Applying Lemma 5 we see that Theorem 3 is a particular case of Theorem 8.

\section{Bibliography}

[1] R. Bělohlávek, Fuzzy Relational Systems. Foundations and Principles, Kluwer, 2002.

[2] U. Bodenhofer, Similarity-based Generalizations of Fuzzy Orderings Preserving the Classical Axioms, International Journal of Uncertainty, Fuzziness and Knowledge Based Systems, Vol. 3, pp. 593-610, 2000.

[3] U. Bodenhofer, F. Klawonn, A Formal Study of Linearity Axioms for Fuzzy Orderings, Fuzzy Sets and Systems, Vol. 145, pp. 323-354, 2004.

[4] S. Gottwald, Fuzzy Sets and Fuzzy Logic, Vieweg, Braunschweig, 1993.

[5] P. Hájek, Methamathematics of Fuzzy Logic, Kluwer, 1998.

[6] U. Höhle, N. Blanchard, Partial Ordering in L-undeterminate sets, Information Sciences, Vol. 35, pp. 135-144, 1985.

[7] E. P. Klement, R. Mesiar, E. Pap, Triangular Norms , Kluwer, 2000.

[8] M. Richter, Revealed Preference Theory, Econometrica, Vol. 34, pp. 635-645, 1966.

[9] I. J. Rudas, J. Fodor, Information Aggregation in Intelligent Systems using Generalized Operators, International Journal of Computers, Communications and Control, Vol. 1, pp. 47-57, 2006. 
[10] E. Szpilrajn, Sur l'Extension de l'Ordre Partiel, Fundamenta Mathematicae, Vol. 16, pp. 386-389, 1930.

[11] L. A. Zadeh, Similarity Relations and Fuzzy Orderings, Information Sciences, Vol. 3, pp. 177-200, 1971.

\author{
Irina Georgescu \\ Academy of Economic Studies \\ Department of Economic Cybernetics \\ Piata Romana No 6, R 70167, Oficiul Postal 22 \\ Bucharest, Romania \\ E-mail: irina.georgescu@csie.ase.ro
}

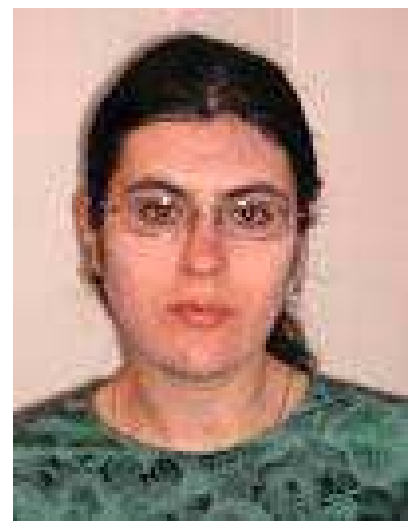

Irina Georgescu received her $\mathrm{PhD}$ in Economics (Information Systems) from Abo Akademi University, Turku, Finland in 2005. Since then she is a teaching assistant at the Department of Economic Cybernetics, Academy of Economic Studies, Bucharest, Romania. Between 2007-2008 she was a postdoctoral researcher at Abo Akademi University, Turku, Finland. She is the author of about 30 scientific publications and of a monograph issued by Springer. Her research interests are mainly in the area of soft computing techniques, consumer theory and social choice and welfare economics. 